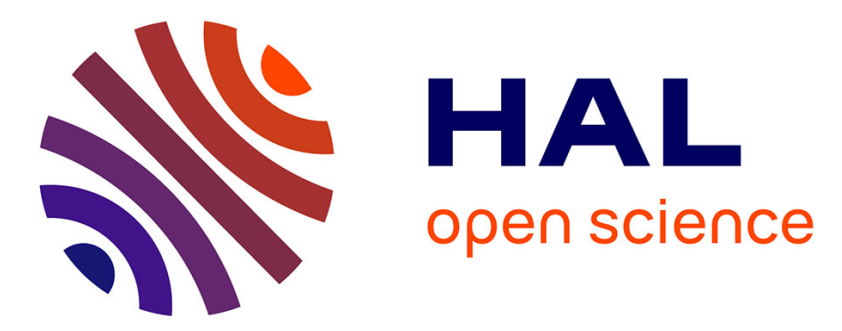

\title{
Infrared image processing for the calorimetric analysis of fatigue phenomena
}

\author{
B. Berthel, Andre Chrysochoos, Bertrand Wattrisse, A. Galtier
}

\section{To cite this version:}

B. Berthel, Andre Chrysochoos, Bertrand Wattrisse, A. Galtier. Infrared image processing for the calorimetric analysis of fatigue phenomena. Experimental Mechanics, 2008, 48 (1), pp.79-90. 10.1007/s11340-007-9092-2 . hal-00572333

\section{HAL Id: hal-00572333 \\ https://hal.science/hal-00572333}

Submitted on 24 Sep 2021

HAL is a multi-disciplinary open access archive for the deposit and dissemination of scientific research documents, whether they are published or not. The documents may come from teaching and research institutions in France or abroad, or from public or private research centers.
L'archive ouverte pluridisciplinaire HAL, est destinée au dépôt et à la diffusion de documents scientifiques de niveau recherche, publiés ou non, émanant des établissements d'enseignement et de recherche français ou étrangers, des laboratoires publics ou privés. 


\title{
Infrared Image Processing for the Calorimetric Analysis of Fatigue Phenomena
}

\author{
B. Berthel • A. Chrysochoos • B. Wattrisse • A. Galtier
}

\begin{abstract}
This paper presents an infrared image processing procedure that was developed to study calorific effects accompanying material fatigue. This method enables us to separately estimate patterns of thermoelastic and dissipative sources. Heat sources were estimated on the basis of partial derivative operators present in a local form of the heat equation by using a set of approximation functions that locally fits the temperature field and takes the spectral properties of the sought sources into account. Numerical examples were used to check the validity of the method and to highlight its capabilities along with its limits. The paper concludes with examples of thermal image processing extracted from fatigue tests performed on a dual-phase steel. The coupling sources were compared to the theoretical predictions induced by a basic thermoelastic model, while the heterogeneous character of the fatigue development was highlighted in terms of dissipation sources.
\end{abstract}

\author{
B. Berthel $(\bowtie) \cdot A$. Chrysochoos $\cdot$ B. Wattrisse \\ Mechanics and Civil Engineering Laboratory, \\ Montpellier II University, \\ CC 48, Place E. Bataillon, \\ 34095 Montpellier Cedex 05, France \\ e-mail: Berthel@1mgc.univ-montp2.fr \\ A. Chrysochoos \\ e-mail: Chryso@lmgc.univ-montp2.fr \\ B. Wattrisse \\ e-mail: Wattrisse@1mgc.univ-montp2.fr \\ B. Berthel · A. Galtier \\ Arcelor Research SA, \\ Voie Romaine BP 30320, \\ 57283 Maizières-lès-Metz, France \\ A. Galtier \\ e-mail: Andre.Galtier@arcelor.com
}

Keywords Fatigue · Dissipation · Thermoelasticity · Infrared imaging $\cdot$ Steel

\section{Introduction}

Characterization of fatigue in materials and mechanical components requires time-consuming and expensive statistical processing of the results of numerous mechanical cyclic tests. Alternative experimental approaches have been developed to rapidly provide reliable fatigue characteristics. Among these, thermal methods, based on an analysis of self-heating during a stepwise loading fatigue test, should be mentioned [1-6]. The authors of these latter studies claimed that the remarkable change in the heating regime, observed at a certain stress range, is empirically related to the fatigue limit of the material. Although realistic estimates of this limit were sometimes obtained, the thermal approach often led to questionable results. Indeed, the physical meaning of the threshold stress evaluated by these thermal methods is not very clear.

In [7] it was shown that the appearance of the persistent slip bands is associated with the loss of linearity of the temperature versus stress range correspondence. It was also shown that the persistent slip band number increases with the stress and appears to be a relevant fatigue indicator.

In previous works, we also underlined that the direct use of temperature as a fatigue indicator is not always reliable because the temperature variation is not intrinsic to the material behaviour [8]. It actually depends on the diffusion properties (material effect) but also on thermal boundary conditions and the heat source distribution (structure effects).

In this work, fatigue phenomena were therefore studied using a calorimetric approach - the goal was to determine the different heat sources accompanying the fatigue test. A 
local form of the heat equation was used to derive heat sources from thermal images provided by an infrared camera. A specific thermal image processing procedure was developed to separately estimate dissipated energy coming from the irreversible variations in the micro-structural defects and the thermoelastic coupling sources induced by reversible thermal expansion of the crystalline network.

In what follows, we first review the expression of the heat diffusion equation and definitions of the different heat sources. We then describe the image processing method together with some numerical simulations in order to illustrate the relevance and limits of heat source computation in the case of noisy under-sampled data. Several experimental results obtained on dual-phase steel are finally shown to underline the promising potential of this calorimetric approach.

\section{Thermomechanical Background}

\section{Balance of Heat and Definition of Sources}

From a thermomechanical standpoint, fatigue is considered as a dissipative quasi-static process. The equilibrium state of each volume material element is then described using a set of $N$ state variables. The chosen variables are: the absolute temperature $T$, the linearized strain tensor $\varepsilon$ (small strain assumption) and $N-2$ scalar components $\alpha_{1}$, $\alpha_{2}, \ldots \alpha_{N-2}$ of vector $\alpha$ which pools the internal variables. These latter describe the macroscopic effects of complex, coupled microstructural phenomena.

Within the Classical Irreversible Thermodynamics framework [9], the local heat diffusion equation can be written as:

$\rho C \dot{T}-\operatorname{div}(K \operatorname{grad} T)=d_{1}+s_{\text {the }}+s_{\text {ic }}+r_{\text {ext }}$

where $\rho$ is the mass density, $C=-T \psi_{, T T}$ the specific heat, $\psi$ the Helmholtz free energy, $K$ the conduction tensor. The left-hand side of equation (1) is a differential operator applied to $T$, while its right-hand side groups all possible heat sources accompanying the deformation process. They are, in turn, the intrinsic dissipation $d_{1}=\sigma: D-\rho \psi_{, \varepsilon}$ : $\dot{\varepsilon}-\rho \psi_{, \alpha} \cdot \dot{\alpha}$, thermomechanical coupling sources that pool the thermoelastic source $s_{\text {the }}=\rho T \psi_{, T \varepsilon}: \dot{\varepsilon}$ and other internal coupling sources $s_{\mathrm{ic}}=\rho T \psi_{T} \alpha \cdot \dot{\alpha}$, and the external volume heat supply $r_{\mathrm{ext}}$. The product $\sigma: D$ stands for the volume deformation energy rate, where $\sigma$ is the Cauchy stress tensor and $D$ the Eulerian strain rate tensor.

Towards an Operational Form of the Heat Equation

The overall heat source can then be theoretically estimated by evaluating the left-hand side of the heat equation. Nevertheless, the only thermal information given by the IR camera is surface temperature fields. The heat source evaluation throughout the specimen gauge part (3D inverse problem) is an ill-posed problem that is impossible to solve without any information about the heat source distribution [10].

The regularizing effects of heat diffusion fortunately limit the thermal gradients throughout the (small) thickness of the specimen (Fig. 1). This effect has already been mentioned in the case of uniformly distributed heat sources within an infinite cylinder [11]. Hereafter we will show that this result can be extended to strongly heterogeneous distributions of heat sources within metallic thin flat samples. In particular, we will establish that thermal data on the surface remain close to the averaged temperature during cyclic tests, as already shown in [12] for monotonous loadings.

This result warranted the use of an integrated form of the heat equation over the sample thickness to estimate the depth-wise averaged heat source distribution. The following hypotheses were also formulated to perform the heat source evaluation: (1) the conduction tensor $K$ is isotropic and the related coefficient $k$ remains constant during the test; (2) parameters $\rho$ and $C$ are material constants, independent of the internal state; (3) the convective terms included in the material time derivation are neglected; (4) the external heat supply $r_{\text {ext }}$ (here only due to radiation heat exchanges) is time-independent. Consequently, the equilibrium temperature field $T_{0}$ verifies $-k \Delta T_{0}=r_{\text {ext }}$.

While temperature variations $\theta=T-T_{0}$ induced by fatigue mechanisms remain small, we admit that they have no influence on the microstructural state. It can therefore be stated that $s_{\mathrm{ic}}=0$.

Under these hypotheses, the local 3D heat equation (1) can be rewritten in the following simplified form:

$\rho C \frac{\partial \theta}{\partial \tau}-k \Delta \theta=d_{1}+s_{\text {the }}$

where $\tau$ is the time and $\Delta$ the Laplacian operator.

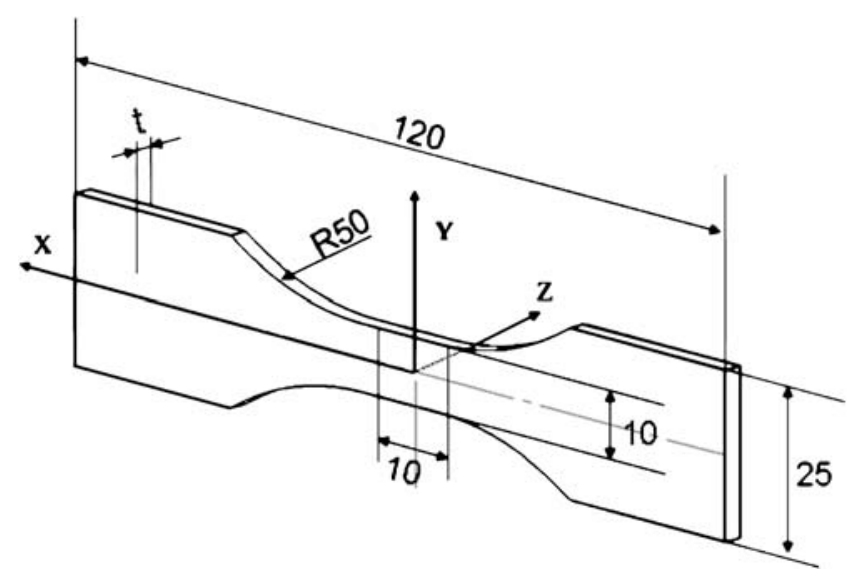

Fig. 1 Shape of the specimen 
The following 2D equation can be obtained

$\frac{\partial \bar{\theta}}{\partial \tau}+\frac{\bar{\theta}}{\tau_{\text {th }}^{2 \mathrm{D}}}-\frac{k}{\rho C}\left(\frac{\partial^{2} \bar{\theta}}{\partial x^{2}}+\frac{\partial^{2} \bar{\theta}}{\partial y^{2}}\right)=\frac{\bar{d}_{1}}{\rho C}+\frac{\bar{s}_{\text {the }}}{\rho C}$

(1) assuming symmetrical linear heat exchanges between the front/back specimen faces and the surroundings $\left(-k \partial T /\left.\partial z\right|_{z= \pm t / 2}= \pm h\left(T-T_{0}\right)\right.$, where $h$ is the heat exchange coefficient by convection and radiation)

(2) integrating equation (2) over the sample thickness $t$

(3) assuming that the thermal image is very close to the depth-wise averaged temperature field $\bar{\theta}(x, y, \tau)=$ $\frac{1}{t} \int_{-t / 2}^{+t / 2} \theta(x, y, z, \tau) \mathrm{d} z$.

In equation (3), $\bar{\theta}$ represents an averaged (2D) distribution and $\tau_{\text {th }}^{2 \mathrm{D}}=\rho C t /(2 h)$ stands for a time constant characterizing perpendicular heat exchanges between front and back specimen faces and the surroundings. This 2D approach has already been successfully used to track localization zones during monotonic tensile tests [12].

\section{Infrared Image Processing}

\section{Principle of the Method}

The determination of heat sources is based on an estimate of the various differential operators of equation (3) applied to temperature variations. The reliability of such estimates depends on the control of several different items: (1) accuracy of the temperature measurements, (2) relevance of the hypotheses leading to equation (3) and especially those concerning the depth-wise averaged temperature $\left(H_{1}\right)$ and the convective terms $\left(H_{2}\right)$, (3) efficiency of the method used to compute the sources on the basis of discrete, noisy thermal data.

\section{Order of Magnitude of Heat Sources and Temperature} Variations

Concerning the last item, we stress that derivation of $s_{\text {the }}$ and $d_{1}$ is the ultimate objective of the image processing. At this level, two main difficulties should be underlined concerning noise amplification in estimating the derivatives and the fact that the intensities of both sources are not in the same order of magnitude. During a fatigue test performed at constant loading frequency $f_{\mathrm{L}}$, constant stress range $\Delta \sigma$ and constant stress ratio $R_{\sigma}$, the temperature variation indeed behaves as shown in Fig. 2 .

In this example, dissipation leads to warming of the specimen up to $3^{\circ} \mathrm{C}$ within $180 \mathrm{~s}$, while the thermoelastic effects generate cyclic temperature variations of about $0.4^{\circ} \mathrm{C}$ every $\frac{1}{2} f_{\mathrm{L}}^{-1} \approx 0.017 \mathrm{~s}([13,14])$. The order of

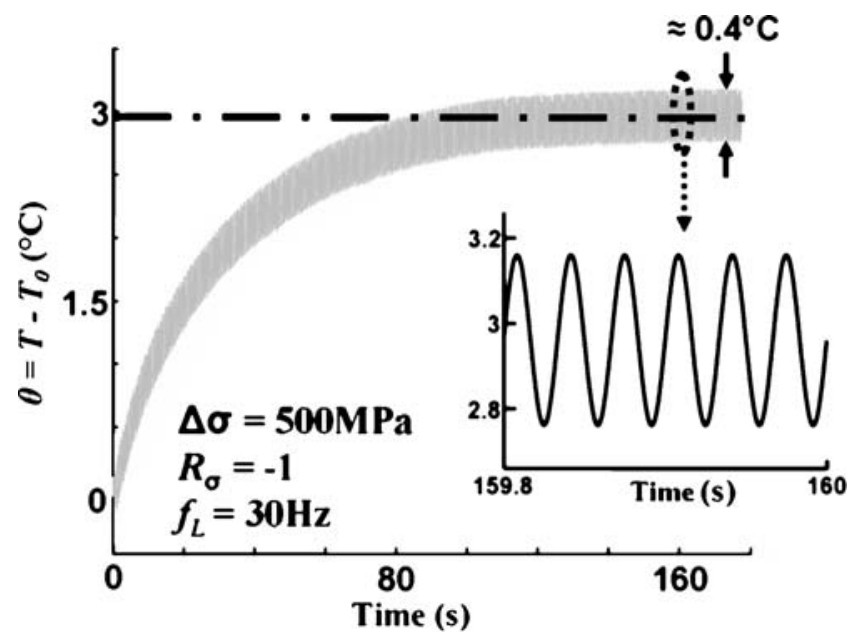

Fig. 2 Typical temperature pattern during a fatigue test

magnitude of both heat sources can be obtained through a differential diffusion equation [17] that can be used when the heat sources are uniformly distributed. This equation reads:

$\frac{\mathrm{d} \bar{\Theta}}{\mathrm{d} \tau}+\frac{\bar{\Theta}}{\tau_{\text {eq }}}=\frac{\bar{d}_{1}}{\rho C}+\frac{\bar{s}_{\text {the }}}{\rho C}$

where $\bar{\Theta}$ is the mean temperature variation captured at the centre of the sample gauge part, and $\tau_{\text {eq }}$ is the overall time constant characterizing all the local heat losses. To roughly match the results of Fig. 2, the thermoelastic source range $\overline{\Delta s}_{\text {the }} / \rho C$ and the mean dissipation per cycle $\widetilde{d}_{1}(x, y) / \rho C=$ $\int_{f^{-1}} f_{L} \bar{d}_{1}(x, y, \tau) \mathrm{d} \tau / \rho C$ are $75^{\circ} \mathrm{C} \mathrm{s}^{-1}$ and $0.1^{\circ} \mathrm{C} \mathrm{s}^{-1}$, respectively. In what follows, $\widetilde{d}_{1}$ refers to the mean dissipation intensity per cycle. Note that the sources divided by $\rho C$ are then expressed in ${ }^{\circ} \mathrm{C} \mathrm{s}^{-1}$. This operation makes it possible to define, for each type of source, an equivalent heating speed associated with a monotonous thermal process in adiabatic conditions and facilitates comparison between the coupling and dissipative sources. The thermal effects and their calorific causes then present "inverse" orders of magnitude. Warming due to dissipation is about 10 -fold greater than the temperature variations induced by thermoelasticity, while the dissipation intensity is several hundredfold lower than the thermoelastic source range. This could be easily explained by the nature of both sources: the dissipation is always positive while the thermoelastic source changes its sign with loading. This difference in order of magnitude underlines the major importance of the quality of thermal measurements in the reliable determination of both types of source.

Camera Calibration

The camera used in this work is a Cedip Jade MWIR 3$5.2 \mu \mathrm{m}$. The focal length of the optical lens is $25 \mathrm{~mm}$. This 
camera is equipped with an $\mathrm{InSb} 320 \times 240$ element detector, cooled at $80 \mathrm{~K}$ with a Stirling device. The lens axis of the camera was kept fixed and held perpendicular to the surface of the specimen during the mechanical tests.

A stable and spatially uniform IR radiation source must be used to perform the camera signal calibration. We therefore used a planar black body SR 80-4A (by CI Systems) with the following main specifications: accuracy $\left(+/-0.008^{\circ} \mathrm{C}\right.$ for $5^{\circ} \mathrm{C}$ temperature amplitude), thermal resolution $\left(\delta T_{R}=\right.$ $0.01^{\circ} \mathrm{C}$ ), and spatial non-uniformity of the target (less than $+/-0.02^{\circ} \mathrm{C}$ ).

The standard calibration is based on homogenization of the detector response, whereas pixel responses are generally heterogeneous. All is done to get a uniform signal of the detector elements when the detector is placed in front of a uniform source (non uniformity correction NUC). This enables the user to identify (and apply) a single calibration law. We already pointed out in [16] that the NUC operation supposes a linear response of each detector element. First, this condition may limit the thermal range used for the calibration or may induce uncontrolled errors if some elements start to behave nonlinearly. Secondly, even if all element responses remain linear, the standard calibration protocol replaces some bad pixels because their responses are far from the mean detector response (bad pixel replacement BPR). In the framework of our thermomechanical applications, this replacement operation may distort the thermal gradient computation by spatially correlating measurement errors. This distortion consequently affects the heat source estimate. These are the main reasons why a new protocol based on individual calibration of detector elements has been proposed. It is possible to shortcut NUC and BPR operations with the IR thermography workstation used in this study. This feature was used to develop an individual pixel calibration based on polynomial fitting of the temperature as a function of the signal $s_{i}$ delivered by the $i$ th element of the detector when the camera is placed in front of the black body source at different temperatures $T^{\mathrm{BB}}$,

$T_{j}^{\mathrm{BB}}=\sum_{p=0}^{P} a_{i p} s_{i j}^{p}$ for $T_{j}^{\mathrm{BB}} \in\left[T_{1}, T_{2}\right]$

The $a_{i p}$ coefficients are derived from a least-squares fit. The signal $s_{i j}$ is the one delivered by the pixel $i$ when the black body is at equilibrium temperature $T_{j}^{\mathrm{BB}}$. The user may first define the degree $P$ of the polynomial fitting function. Generally polynomials of degree $P \geq 5$ are chosen. The user defines a number $J$ of equilibrium thermal states $T_{j}^{\mathrm{BB}}$ distributed between $T_{1}$ and $T_{2}$.

When the difference between the temperature of a pixel, deduced from the calibration, and the black body temperature is greater than a given threshold (equal here to $40 \mathrm{mK}$ ), it is considered to be defective. Typically, $0.3 \%$ of bad pixels are identified on our camera. They are rather well distributed over the detector surface. Nevertheless several bad pixel packs could be observed.

Naturally, other parameters may degrade the quality of the temperature measurements (thermal drift, parasitic radiation emanating from the lenses, etc). Readers interested in these tricky metrological issues can refer to $[14,15]$. To conclude this section, we estimated that the peak-to-peak thermal noise was about $200 \mathrm{mK}$ (i.e. before data filtering) and the range of the thermal noise dropped to $2 \mathrm{mK}$ for standard filtering parameters (see "Lowering the thermal noise" section). Moreover, the order of magnitude of the spatial resolution was about $0.16 \mathrm{~mm}$ and the temporal resolution was considered to be equal to the $0.05 \mathrm{~s}$ acquisition period used in the fatigue tests.

Checking Hypotheses $H_{1}$ and $H_{2}$

Equation (3) is based on the assumption that: (1) the surface temperature is very close to $\bar{\theta},(2)$ the convective terms remain negligible.

\section{Hypothesis $H_{1}$}

To check the relevance of $H_{1}$, even in unfavourable cases, academic distributions of heat sources were analyzed. Only dissipation sources were considered in the 3D finite difference simulations. Indeed, plane stress states can be assumed in the case of tests on thin flat samples. For linear thermoelastic behaviour, $s_{\text {the, }}$ being related to the trace rate of the stress tensor, remains constant throughout the sample thickness. In such a situation, it is easy to verify that the temperature remains practically constant in the $z$-direction when we consider the thermal boundary conditions associated with natural convection and low radiation.

We thus only considered a distribution of dissipation intensity where $d_{1}$ was set at zero over part of the sample thickness and constant in the $z$-direction over the complementary part. This latter was characterized by parameter $h$, defined by:

$$
\begin{aligned}
-1 & \leq h=-\frac{z_{1}+z_{2}}{t} \\
& \leq 1 \text { with }\left\{\begin{array} { c } 
{ - t / 2 < z _ { 1 } \leq t / 2 } \\
{ z _ { 2 } = - t / 2 }
\end{array} \text { or } \left\{\begin{array}{c}
-t / 2 \leq z_{2}<t / 2 \\
z_{1}=t / 2
\end{array}\right.\right.
\end{aligned}
$$

where $t=2.6 \mathrm{~mm}$ and $z_{1}$ and $z_{2}$ are the parameters shown in Fig. 3(a). If we define $\xi(z)$ as $\xi(z)=1$ if $z \in] z_{1}, z_{2}[$ and 0 outside, the dissipation throughout the volume took the following form $d_{1}(x, y, z, \tau)=\bar{d}_{1}(x, y)\left|\frac{\pi t}{2\left(z_{2}-z_{1}\right)}\right|\left|\sin \left(2 \pi f_{\mathrm{L}} \tau\right)\right|$ $\xi(z)$, in the numerical computations, where $d_{1}$ is a random distribution. 
Fig. 3 Checking $H_{1}$ : (a) basic sketch of dissipative sources distribution (b) maximal absolute value of the difference between the depthwise averaged temperature and the surface temperature

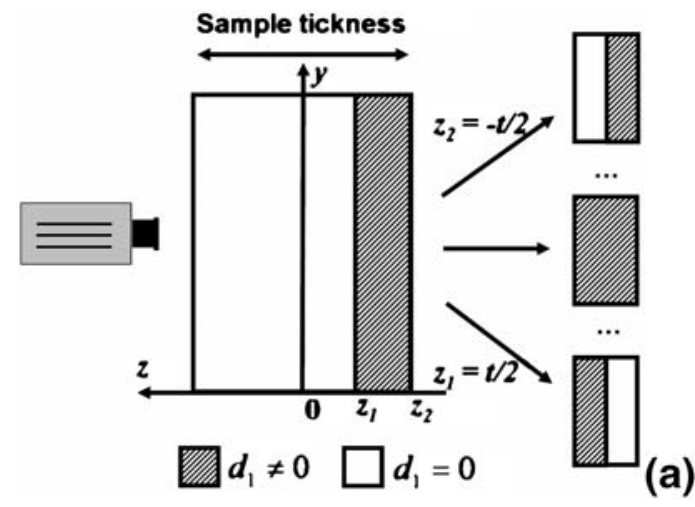

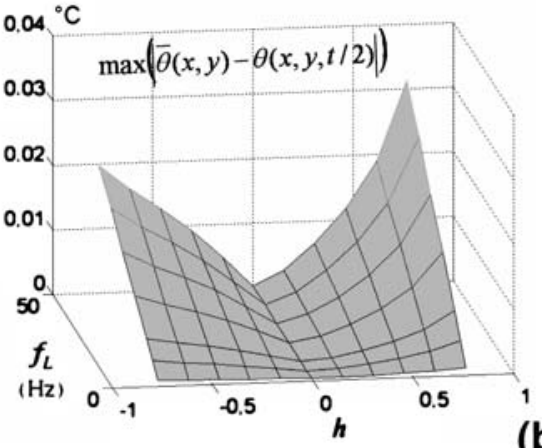

(b)

Simulations were performed at different $f_{\mathrm{L}}$. Fourier's linear boundary conditions were considered in the $3 \mathrm{D}$ finite difference computations. The heat exchange coefficients were chosen close to those identified experimentally. The depth-wise averaged dissipation was set at a constant value (for a given $f_{\mathrm{L}}$ in order to roughly get a constant temperature variation level whatever the value of $h$.

Figure 3(b) shows that the maximal absolute value of the difference between the average temperatures over the thickness and the surface temperatures. As expected, an increase in the difference with $f_{\mathrm{L}}$ and $h$ can be observed. However, for all couples $\left(f_{\mathrm{L}}, h\right)$, the difference remains less than $0.04^{\circ} \mathrm{C}$ and is then within the same order of magnitude as the temperature accuracy. Surface temperature provided by the infrared camera could be considered as the depthwise averaged temperature.

\section{Hypothesis $\mathrm{H}_{2}$}

Hypothesis $\mathrm{H}_{2}$ allows us to write a form of the heat equation involving temperature fields only. This implies that heat losses by matter convection are negligible. These convective terms can be written as:

$\dot{T}=\frac{\partial T}{\partial \tau}+\underbrace{v \cdot \operatorname{grad} T}_{\text {convective term }}$

where $v$ is the velocity vector. Estimating the time derivative then requires the knowledge of the velocity field. In what follows, we show that the heat transfer by matter convection remains negligible in the framework of our fatigue tests.

To verify the relevance of $\mathrm{H}_{2}$, we analysed situations where displacement and temperature fields are known. The comment about the dissipation intensity compared to the range of the thermoelastic sources $\left(10^{-3} \leq \widetilde{d}_{1} / \overline{\Delta s}_{\text {the }} \leq\right.$ $10^{-2}$ ) led us to consider coupled thermoelastic problems where a very slight dissipation is randomly distributed, assuming that stress and strain patterns are not too disturbed by irreversible mechanisms.

In the following, results concerning a cyclic tensile and bending test are shown. The gauge part of the specimen is now considered as a beam whose length, width and thickness are, respectively, denoted by $L, w, t$, as shown in Fig. 4.

Top and bottom sides of the beam are stress free; the left side is subjected to the resulting forces $P$ and $F$, while the right side is clamped. In the frame of reference defined in Fig. 7, the matrix of the stress tensor can be written as:

$$
\begin{gathered}
\sigma=\left(\begin{array}{ccc}
\frac{12 P}{t w^{3}} x y+\frac{F}{w t} & \frac{6 P}{4 t w}\left(1-\frac{4 y^{2}}{w^{2}}\right) & 0 \\
\frac{6 P}{4 t w}\left(1-\frac{4 y^{2}}{w^{2}}\right) & 0 & 0 \\
0 & 0 & 0
\end{array}\right) \\
\cos \left(2 \pi f_{L} \tau\right) \text { where }\left\{\begin{array}{c}
0 \leq \mathrm{x} \leq \mathrm{L} \\
-\frac{\mathrm{w}}{2} \leq \mathrm{y} \leq \frac{\mathrm{w}}{2} \\
0 \leq \tau \leq \tau \mathrm{M}
\end{array}\right.
\end{gathered}
$$

By using the linear isotropic elastic law and neglecting the very low thermal strain, we can derive the strain matrix. The use of this latter and that of the displacement boundary conditions classically gave us the displacement field:

$\left\{\begin{array}{l}u_{x}=\left[\begin{array}{l}\left.\frac{12 P}{E E w^{3}}\left(x^{2} y-\frac{1}{3}(2+\nu) y^{2}+\left((1+\nu) \frac{w}{2}-L^{2}\right) y\right)\right] \\ \frac{F L}{E w t}(x-1)\end{array}\right] \cos \left(2 \pi f_{L} \tau\right) \\ u_{y}=\left[\frac{12 P}{E t w^{3}}\left(-\nu x^{2} y-x^{2}+L^{2} x-\frac{2 L^{3}}{3}\right)-\frac{\nu F L}{E w t} y\right] \cos \left(2 \pi f_{L} \tau\right) \\ u_{z}=\left[-\frac{\nu 6 P}{E t w^{3}} x y z-\frac{\nu F L}{E w t} z\right] \cos \left(2 \pi f_{L} \tau\right)\end{array}\right.$

where $E$ is the Young's modulus and $v$ is the Poisson's ratio.

With equation (7), the thermoelastic source range can be approximated by [17]:

$\Delta s_{\text {the }}=\lambda_{\text {th }} \Delta(T \operatorname{tr} \dot{\sigma}) \approx 4 \pi f_{\mathrm{L}} \lambda_{\text {th }} T_{0}\left(\frac{12 P}{t w^{3}} x y+\frac{F}{t w}\right)$

where $\lambda_{\text {th }}$ is the linear thermal expansion coefficient.

We superimposed 3D random distributions of slight dissipative sources. The randomization was chosen to generate thermal gradients induced by the possible hetero- 


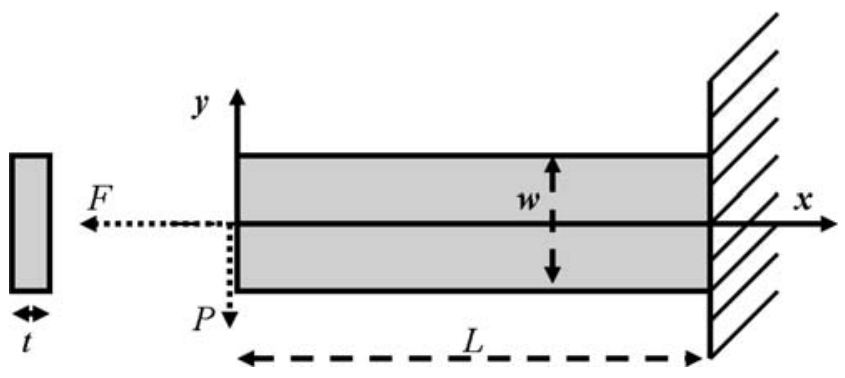

Fig. 4 Tensile and bending test

geneous development of the fatigue mechanisms. The distribution took into account the existence of high and low dissipation zones, as suggested by the experiments (see Figs. 12 and 13). We also considered dissipations having a constant mean value per cycle $\widetilde{d}_{1}$. This property must translate the very slow variation of fatigue mechanisms during a long period of the specimen life under fixed loading conditions. This last property of fatigue phenomena will be shown in the section dealing with the experimental results. The velocity field $v$ was derived from equation (8) to compute $\phi=|v \cdot \operatorname{grad} T|$ and determine its maximum.

In Fig. 5, we gathered the maximal values of the convective terms for a fixed loading frequency $\left(f_{\mathrm{L}}=30 \mathrm{~Hz}\right)$, different loading conditions $(F=5 \mathrm{kN}, P=0,40, \ldots, 200 \mathrm{~N})$ and for a random distribution of dissipation, such that $\widetilde{d}_{1} / \rho C \in[0,0.5]^{\circ} \mathrm{Cs}^{-1}$.

The abscissa axis indicates the level of stress (or thermoelastic source) variations throughout the beam. For instance, the term $\delta \Delta s_{\text {the }}$, that quantifies the heterogeneous distribution of thermoelastic sources over the sample gauge part, is defined by:

$\delta\left(\Delta s_{\text {the }}\right)=\max _{x, y}\left(\Delta s_{\text {the }}\right)-\min _{x, y}\left(\Delta s_{\text {the }}\right)$

For a homogeneous tensile test $\delta \Delta s_{\text {the }}=0$. The maxima of $\phi$ were computed considering about 100 successive cycles. In this example, numerical simulations showed that the minimal value $6.6 \times 10^{-3}{ }^{\circ} \mathrm{C} \mathrm{s}^{-1}$, obtained for homogenous thermoelastic sources, came essentially from the heterogeneity of dissipation fields $\left(6.5 \times 10^{-3}{ }^{\circ} \mathrm{C} \mathrm{s}^{-1}\right)$. The maximal value of $\phi$, obtained for the highest intensity of the bending load $(P=400 \mathrm{~N})$, was about $10^{-2}{ }^{\circ} \mathrm{C} \mathrm{s}^{-1}$. This value is less than the smallest dissipation intensity that can be reasonably detected by the method, as can be seen hereafter (see Fig. 7). Consequently, the convective terms were neglected in the computation of the different heat sources.

\section{Heat Source Computation}

Construction of the heat source distribution via equation (3) requires the evaluation of partial derivative operators applied to noisy digital signals. To compute reliable estimates of heat sources, it is then necessary to reduce the noise amplitude without modifying the spatial and temporal thermal gradients.

\section{Set of approximation functions}

Among several possible methods, a special local leastsquares fitting of the thermal signal was considered in this work. The temperature approximation functions account for the spectral properties of the underlying heat sources. Moreover, the linearity of equation (3) and that of the respective boundary conditions enabled us to separately analyze the influence of thermoelastic and dissipative heat sources. Moreover, within the linear thermoelasticity framework, it is easy to verify that:

- The thermoelastic source has the same frequency spectrum as the stress signal [cf. equation (9)];

- The variation in the thermoelastic energy $w_{\text {the }}$ vanishes at the end of each loading cycle of period $f_{\mathrm{L}}^{-1}$, so we get:

$$
w_{\text {the }}=\int_{f_{\mathrm{L}}^{-1}} s_{\text {the }} \mathrm{d} \tau=0
$$

Regarding the dissipative effects, we have considered the dissipation averaged over a whole number $n$ of complete cycles (e.g. $n \sim$ number of cycles per second):

$\widetilde{d}_{1}=\int_{n f_{L}^{-1}} n^{-1} f_{L} \bar{d}_{1} \mathrm{~d} \tau$.

The new variable $\widetilde{d}_{1}$ is sufficient to characterize the slow changes of the material microstructure due to fatigue

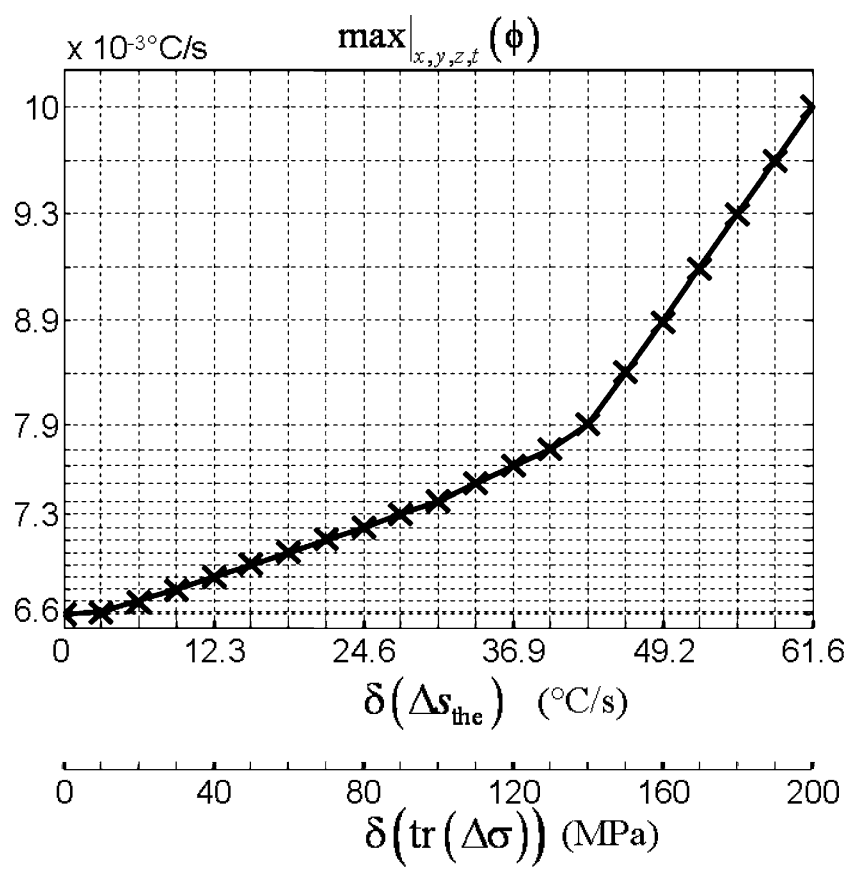

Fig. 5 Maximal intensity of the convective term 

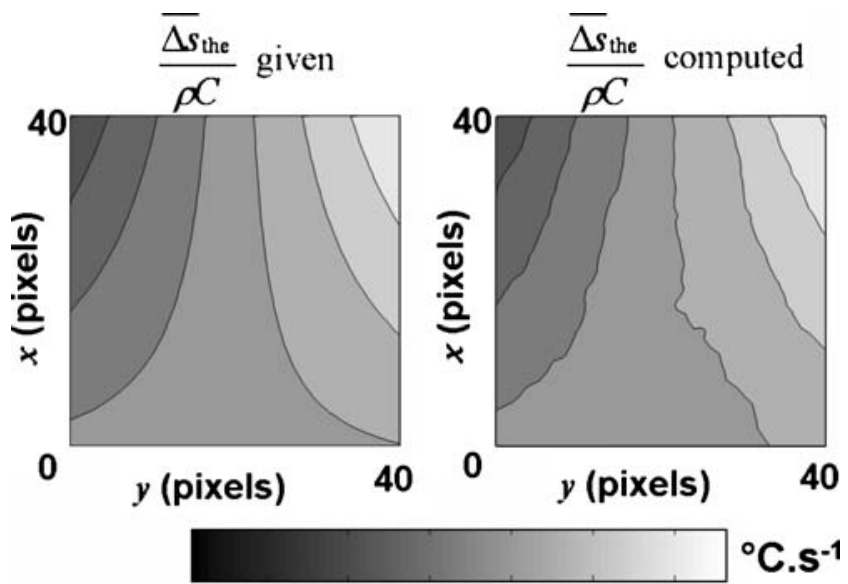

$\begin{array}{llllll}30 & 40 & 50 & 60 & 70 & 80\end{array}$

Fig. 6 Example of a comparison between given and computed patterns

phenomena. The average dissipation per cycle $\widetilde{d}_{1}$ is thus a positive heat source whose spectrum is limited to very low frequencies.

Henceforth by denoting $\overline{\Delta s}_{\text {the }}$ as the range of the thermoelastic source averaged over $n$ cycles, the aim of the image processing is to separately assess $\overline{\Delta s}_{\text {the }}$ and $\widetilde{d}_{1}$.

The local fitting function $\theta^{\text {fit }}$ of the temperature charts is chosen in the same way as in [17]:

$$
\begin{aligned}
\theta^{\mathrm{fit}}(x, y, \tau)= & p_{1}(x, y) \tau+p_{2}(x, y) \\
& +p_{3}(x, y) \cos \left(2 \pi f_{\mathrm{L}} \tau\right) \\
& +p_{4}(x, y) \sin \left(2 \pi f_{\mathrm{L}} \tau\right)
\end{aligned}
$$

where the trigonometric time functions describe the periodic part of the thermoelastic effects while the linear time function takes transient effects due to heat losses, dissipative heating and possible drifts in the equilibrium temperature into account.

The functions $p_{i}(x, y), i=1, \ldots, 4$, are second order polynomials in $x$ and $y$. These polynomials enabled us to account for the possible spatial heterogeneity of the source patterns.
By denoting $P_{1}, \ldots, P_{24}$ as the fitting parameters, the corresponding expressions of $\overline{\Delta s}_{\text {the }}$ and $\widetilde{d}_{1}$. are, respectively:

$\overline{\Delta s}_{\text {the }}=2 \sqrt{\frac{\left(\begin{array}{l}P_{1} \omega \frac{I^{2}}{N_{x}^{g}}+P_{5} \omega \frac{J^{2}}{N_{y}^{g}}+P_{21} \omega \\ +\frac{I}{\tau_{t h}}\left(P_{2} \frac{I^{2}}{N_{x}^{g}}+P_{6} \frac{J^{2}}{N_{y}^{g}}+P_{22}\right) \\ -\frac{2 k}{\rho C}\left(\frac{P_{2}}{\Delta x^{2}}+\frac{P_{6}}{\Delta y^{2}}\right)\end{array}\right)^{2}}{+\left(\begin{array}{l}-P_{2} \omega \frac{I^{2}}{N_{x}^{g}}-P_{6} \omega \frac{J^{2}}{N_{y}^{g}}-P_{22} \omega \\ +\frac{I}{\tau_{t h}^{2 d D}}\left(P_{1} \frac{I^{2}}{N_{x}^{g}}+P_{5} \frac{J^{2}}{N_{y}^{g}}+P_{21}\right) \\ -\frac{2 k}{\rho C}\left(\frac{P_{1}}{\Delta x^{2}}+\frac{P_{5}}{\Delta y^{2}}\right)\end{array}\right)^{2}}}$

$$
\begin{aligned}
\frac{\widetilde{d}_{1}}{\rho C}= & \left(\frac{P_{3}}{\Delta \tau}+\frac{P_{4}}{\tau_{t h}^{2 D}}\right) \frac{I^{2}}{N_{x}^{g}}+\left(\frac{P_{7}}{\Delta \tau}+\frac{P_{8}}{\tau_{t h}^{2 D}}\right) \frac{J^{2}}{N_{y}^{g}} \\
& +\left(\frac{P_{23}}{\Delta \tau}+\frac{P_{24}}{\tau_{t h}^{2 D}}\right)-\frac{2 k}{\rho C}\left(\frac{P_{4}}{\Delta x^{2}}+\frac{P_{8}}{\Delta y^{2}}\right)
\end{aligned}
$$

where $N_{x}^{g}=2 N_{x}+1$ et $N_{y}^{g}=2 N_{y}+1$ are the number of pixels of the fitting window, $I^{2}=\sum_{i=-N_{x}}^{i=N_{x}} i^{2}$ et $J^{2}=$ $\sum_{i=-N_{y}}^{i=N_{y}} j^{2}, \Delta x$ and $\Delta y$ are the space resolutions, $\Delta \tau$ is the time-step associated with the frame-rate (i.e. the sampling frequency $f_{\mathrm{S}}$ ), and $\omega=f_{\mathrm{L}} / f_{\mathrm{S}}$.

\section{Lowering the thermal noise}

The efficiency of the local fitting of the temperature field can be estimated by considering the temperature difference $\delta \theta_{i j k}$ between the noisy measurements $\theta_{i j k}^{\exp }=\theta^{\exp }\left(x_{i}, y_{j}, \tau_{k}\right)$ and the fitted values $\theta_{i j k}^{\mathrm{fit}}=\theta^{\mathrm{fit}}\left(x_{i}, y_{j}, \tau_{k}\right)$. Let us take, for example, the following standard situation where the approximation data groups $(21 \times 21)$ pixels $\times 57$ frames $(57$ means $3 \mathrm{~s}$ of recording at $f_{\mathrm{S}}=19 \mathrm{~Hz}$ ). For such an example, the mean absolute error $\bar{E}_{\delta \theta}=\langle\delta \theta\rangle$ is less than $10^{-12}{ }^{\circ} \mathrm{C}$ while the standard deviation $\sigma \delta \theta$ is about $0.030^{\circ} \mathrm{C}$. These estimates were done by using a set of experimental noisy thermal data extracted from a real fatigue test. Besides, the
Fig. 7 Comparison between given and computed dissipation intensity profiles (a) $\mathrm{O} x$ profile, (b) Oy profile
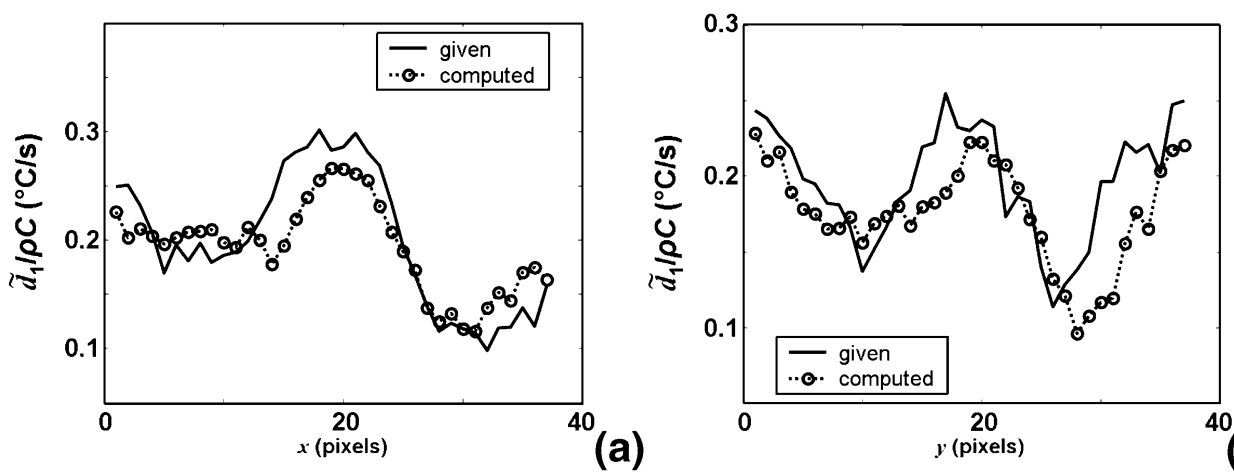

(b) 
Fig. 8 Comparison between given and computed dissipation intensity profiles (a) $\mathrm{O} x$ profile, (b) $\mathrm{O} y$ profile
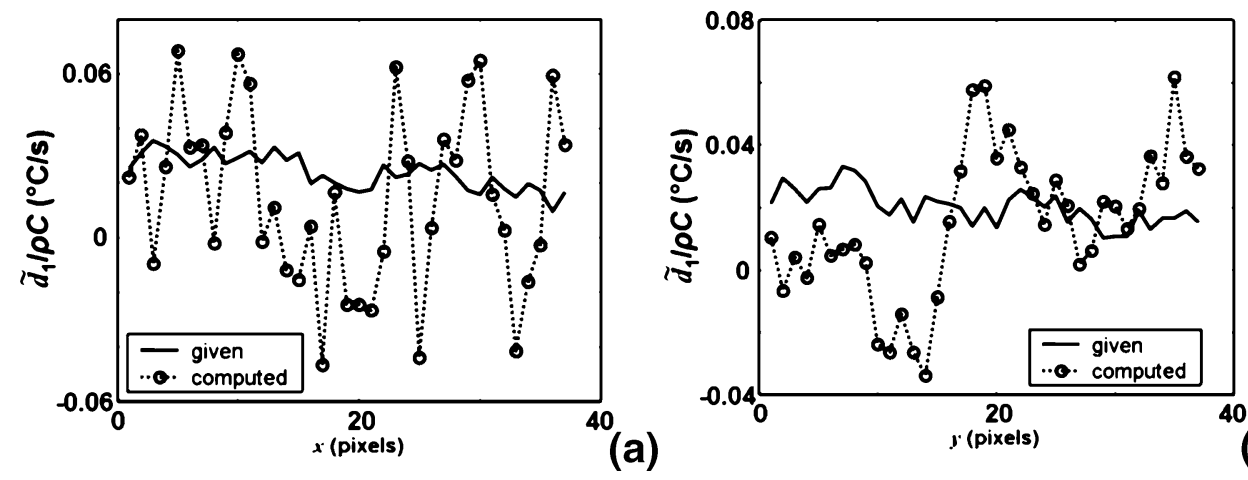

noise in the infrared measurements was characterized by a centred Gaussian distribution with a standard deviation of $0.027^{\circ} \mathrm{C}$. These results show that the approximation function set is convenient to correctly construct a denoised temperature signal.

\section{Checking the heat source computation}

The efficiency of the fitting must also be checked in terms of heat source computation, with this latter being mainly related to the temperature derivatives. Therefore, we considered once again the thermal images obtained during the tension and bending test simulations. Let us remind that for such tests, $s_{\text {the }}$ is constant throughout the thickness (plane stress) and $d_{1}$ is randomly distributed. Noisy data recorded with the camera were superimposed on the computed images.

In Figs. 6 and 7, comparisons between given and computed heat sources are proposed. The following loading parameters were taken into account: $P=0.4 \mathrm{kN}, F=5 \mathrm{kN}, f_{\mathrm{L}}=$ $30 \mathrm{~Hz}$. Figure 9 shows the pattern of $\overline{\Delta s}_{\text {the }}\left(x, y, \tau_{\mathrm{M}} / 2\right)$ captured at the half-period $\tau_{\mathrm{M}} / 2$ of the virtual test and the corresponding pattern of $\overline{\Delta s}_{\text {the }}$ derived from the image processing.

The mean and standard deviation associated with the difference between the given and computed values of the thermoelastic source ranges are, respectively $-1.5^{\circ} \mathrm{C} \mathrm{s}^{-1}$ and $0.023{ }^{\circ} \mathrm{C} \mathrm{s}^{-1}$.

In Fig. 7(a) and (b), the given profiles of $\tilde{d}_{1}(x, y=$ $\left.L / 2, \tau_{\mathrm{M}} / 2\right)$ and $\widetilde{d}_{1}\left(x=w / 2, y, \tau_{\mathrm{M}} / 2\right)$, captured in the middle of the sample width $w / 2$ and length $L / 2$ at the half-period $\tau_{\mathrm{M}} / 2$ of the virtual test, are, respectively, compared to the computed profiles provided by the image processing. The mean and standard deviation associated with the difference between given and computed values of dissipation intensities are, respectively, $28.10^{-4}{ }^{\circ} \mathrm{C} \mathrm{s}^{-1}$ and $64.10^{-4}{ }^{\circ} \mathrm{C} \mathrm{s}^{-1}$ for the whole image.

Despite the different order of magnitude of both source types, the data processing can then quantitatively estimate the amplitudes of the thermoelastic sources and the intensities of the mean dissipation per cycle. The method may naturally become unstable and fail for smaller dissipation intensities, noisier thermal data or too low sampling frequencies.

To illustrate these limits, we chose an example with the same previous conditions, but with 10 -fold smaller dissipation intensities. The method gives corrupted dissipation amounts, as indicated in Fig. 8 where some negative values can be observed, which is physically unacceptable.

Another kind of example shows the influence of a possible surface effect. Indeed fatigue mechanisms may preferentially develop near the specimen surface (persistent
Fig. 9 Checking the image processing for different distributions of $\widehat{d}_{1}(x, y, z)$
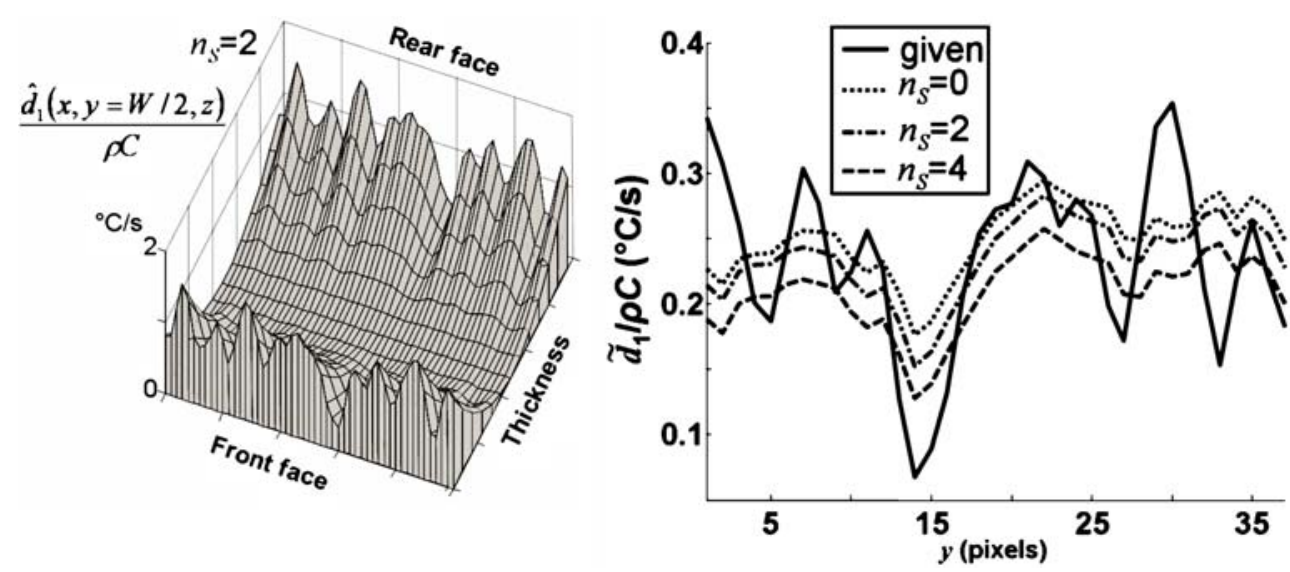
Table 1 Thermophysical properties of DP600

\begin{tabular}{llll}
\hline$\alpha_{d}\left(10^{-6 \circ} \mathrm{C}^{-1}\right)$ & $\rho\left(\mathrm{kg} \mathrm{m}^{-3}\right)$ & $C\left(\mathrm{~J} \mathrm{~kg}^{-1}{ }^{\circ} \mathrm{C}^{-1}\right)$ & $K\left(\mathrm{~W} \mathrm{~m}^{-1}{ }^{\circ} \mathrm{C}^{-1}\right)$ \\
\hline $10-11$ & 7800 & 460 & 64
\end{tabular}

slip bands). It was thus interesting to test distributions where the dissipations were concentrated near the surfaces. A power law was chosen to weight the random distribution over the thickness:

$$
\begin{aligned}
d_{1}(x, y, z, \tau) & =\widetilde{d}_{1}(x, y)\left|\frac{2 z}{t}\right|^{n_{S}} \frac{\pi\left(n_{S}+1\right)\left|\sin \left(2 \pi f_{\mathrm{L}} \tau\right)\right|}{2} \\
& =\widehat{d}_{1}(x, y, z)\left|\sin \left(2 \pi f_{\mathrm{L}} \tau\right)\right|
\end{aligned}
$$

In equation (15) the exponent $n_{\mathrm{s}}$ controls the surface effects [Fig. 12(a)]. This law was constructed to verify:

$\frac{f_{\mathrm{L}}}{t} \int_{f_{\mathrm{L}}^{-1}} \int_{-t / 2}^{+t / 2} d_{1}(x, y, z, \tau) \mathrm{d} z \mathrm{~d} \tau=\widetilde{d}_{1}(x, y), \forall n_{S}$,

to impose a warming level approximately constant for one distribution to another and facilitate the comparative analysis.

In Fig. 9, comparisons between given and computed dissipative sources for different values of $n_{\mathrm{S}}$ are proposed. The profiles of $\widetilde{d}_{1}\left(x=W / 2, y, \tau_{\mathrm{M}} / 2\right)$ captured in the middle of the sample width $W / 2$ at the half-period $\tau_{\mathrm{M}} / 2$ of the virtual test is plotted. When $n_{\mathrm{S}}$ increases, the dissipation estimates slightly decrease but continue to reproduce the global trends of the given dissipative sources. These results may be related to the observations made during the validity check of hypothesis $H_{1}$, where heat source distributions were chosen with a constant depth-wise intensity. These distributions were, however, more academic (piece-wise constant throughout the thickness, uniform over the specimen plane).

Some Comments on the IR Image Processing

The previous sections aimed at showing the relevance of the method developed to separately estimate the dissipation and coupling sources induced by high cycle fatigue. We first briefly recalled that a fine pixel calibration was necessary to correctly extract the small dissipation shrouded in large thermoelastic effects ("Camera Calibration" sec- tion). We then verified that the use of surface temperature provided by the IR camera was warranted by the diffusion mechanisms to compute depth-wise averaged sources $\left(H_{1}\right)$. We also checked that the depth-wise averaged heat source may be computed by neglecting the matter convection $\left(\mathrm{H}_{2}\right)$. The separate estimate of both source types was founded on the linearity of the heat equation and that of the boundary conditions. The temperature fields were then filtered using a special function set accounting for the spectral properties of both source types [equation (12)]. The computation of heat sources was finally checked using a series of numerical benchmark tests in close to experimental conditions.

In the following, the image processing is applied to various experimental fatigue tests to illustrate the potential of this new use of IR data and to highlight the great interest of the calorimetric results.

\section{Application Examples}

The developed image processing then allows us to analyse noisy thermal images provided by the IR camera for a wide range of experimental conditions. In what follows, some properties of thermoelastic source amplitude and mean dissipation during high-cycle fatigue tests performed on a dual phase 600 steel grade are briefly presented. These results do not represent a complete and systematic study of the fatigue behaviour of DP600 steel. As mentioned above, they simply aim at highlighting the interest of the IR image processing presented in this paper.

\section{Material}

The material under examination is DP600 steel produced by Arcelor (Dual Phase Carbon Steel). Thin flat specimens were used with a gauge part of $L=10 \mathrm{~mm}, w=10 \mathrm{~mm}$ and $t=2.6 \mathrm{~mm}$. This material is a hot-rolled steel grade containing ferrite and martensite. It is composed of $0.074 \mathrm{C}$, $0.84 \mathrm{Mn}, 0.038 \mathrm{P}, 0.002 \mathrm{~S}, 0.217 \mathrm{Si}, 0.04 \mathrm{Al}, 0.702 \mathrm{Cr}$, and $0.005 \mathrm{~N}$ (in wt.\%). The thermophysical properties are given in Table 1. Table 2 presents the mechanical properties of DP600: the Young's modulus $E$, the yield strength $\sigma_{y} 0.02$, the ultimate strength $\sigma_{u}$ and the fatigue limits $\Delta \sigma_{\infty}$ (obtained at $2 \times 10^{6}$ cycles) expressed in terms of stress range $\Delta \sigma$ for stress ratio $R_{\sigma}=\sigma_{\min } / \sigma_{\max }$ equal to -1 and 0 , respectively.

Table 2 Mechanical properties of DP60

\begin{tabular}{lllll}
\hline$E(\mathrm{MPa})$ & $\sigma_{y 0.02}(\mathrm{MPa})$ & $\sigma_{u}(\mathrm{MPa})$ & $\Delta \sigma_{\infty}(M P a) R_{\sigma}=0$ & $\Delta \sigma_{\infty}(M P a) R_{\sigma}=-1$ \\
\hline 213000 & 360 & 611 & $464 \pm 15^{*}$ & $526 \pm 5^{*}$ \\
\hline
\end{tabular}

*Standard deviation 
Fig. 10 Patterns of $\overline{\Delta s}_{\text {the }}(x, y)$ for $f_{\mathrm{L}}=50 \mathrm{~Hz}, \mathrm{R}_{\sigma}=0$, for different values of $\Delta_{\sigma}$ and $t$ :
(a) $\Delta_{\sigma}=230 \mathrm{MPa}, \mathrm{t}=54 \mathrm{~s}$,
(b) $\Delta_{\mathrm{\sigma}}=346 \mathrm{MPa}, \mathrm{t}=54 \mathrm{~s}$,
(c) $\Delta_{\sigma}=460 \mathrm{MPa}, \mathrm{t}=54 \mathrm{~s}$,
(d) $\Delta_{\mathrm{\sigma}}=460 \mathrm{MPa}, \mathrm{t}=270 \mathrm{~s}$

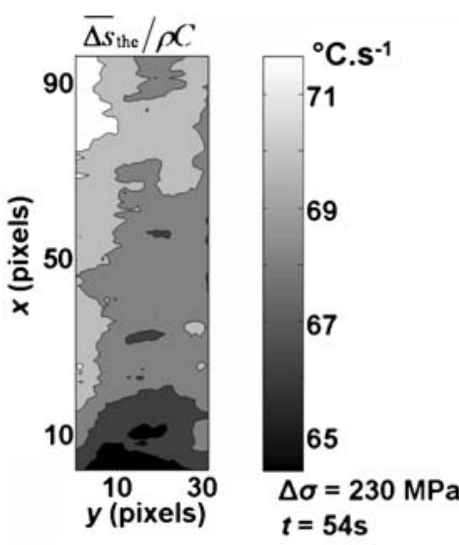

(a)

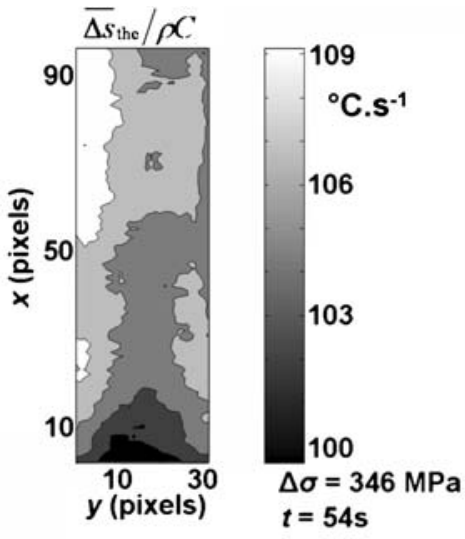

(b)
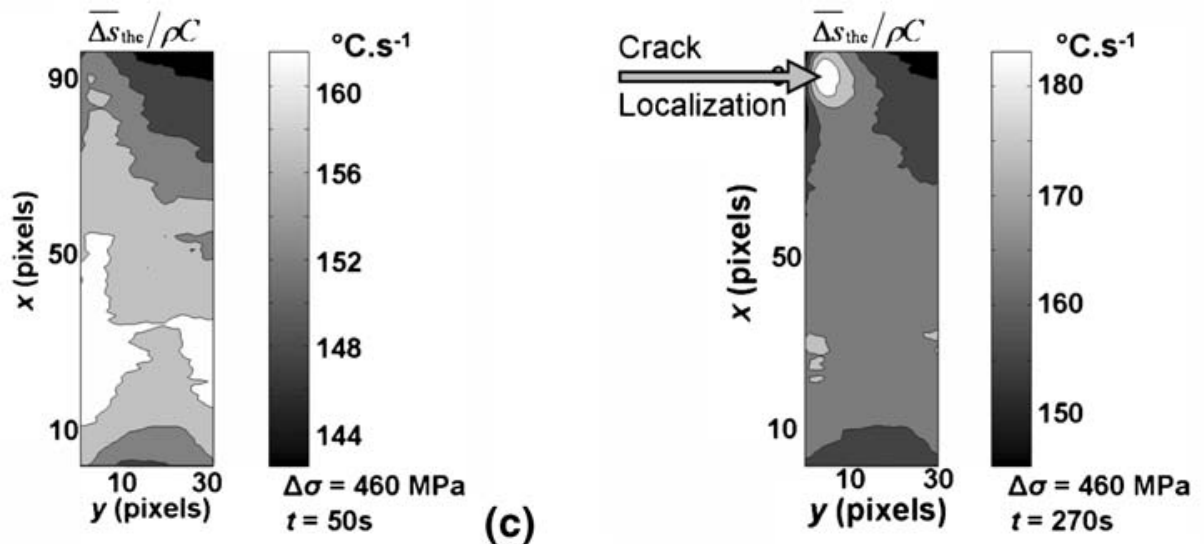

(d)

\section{Testing}

Within the above-mentioned limits imposed by the specimen geometry and the machine capacity, it was possible to carry out load-controlled fatigue tests up to the fatigue limit for the two load ratios, $R_{\sigma}=0$ and $R_{\sigma}=-1$, with the loading being a pure sine until $50 \mathrm{~Hz}$. We checked the monochromatic character of the loading by analysing the frequency spectrum of the load cell signal. The fatigue tests were made of blocks of a limited number of cycles $(\leq 20,000)$, compatible with the finite RAM capacities of the IR device. Each block was performed at constant loading frequency $f_{\mathrm{L}}\left(3 \leq f_{\mathrm{L}} \leq 50 \mathrm{~Hz}\right)$, constant load ratio $R_{\sigma}$, and constant stress range $\Delta \sigma$. At the end of each loading block, the next block is started only after the thermal equilibrium has been reached. For a single specimen, all loading parameters may vary between blocks.

Properties of $\overline{\Delta s}_{\text {the }}$

Until crack onset, we observed in the gage area quasiuniform thermoelastic source amplitude patterns as expected for a simple tension-compression test. Besides, the experiments showed that $\overline{\Delta s}_{\text {the }}$ increased linearly with $\Delta \sigma$ and $f_{\mathrm{L}}$ as predicted by linear thermoelasticity. Such results were already widely presented and discussed in [17]. We high- light hereafter the capabilities of the method in the case of more complex loadings. In the test shown below, the specimen was slightly misaligned in order to induce slight gradients in the thermoelastic source distribution. The test involved loading blocks of 20,000 cycles performed at different $\Delta \sigma$ with $R \sigma=0$ and $f_{\mathrm{L}}=50 \mathrm{~Hz}$.

Despite the out-of-axis loading, Fig. 11 shows that the $\min / \max$ values of $\overline{\Delta S}_{\text {the }}$ increase linearly with $\Delta \sigma$ until the yield stress is reached $\left(\sigma_{y}=360 \mathrm{MPa}\right)$. The slopes of both curves are different because the stress pattern is not homogenous. This means that the "elastic" stress field changes with $\Delta \sigma$ in a homothetic way, so the overall stress

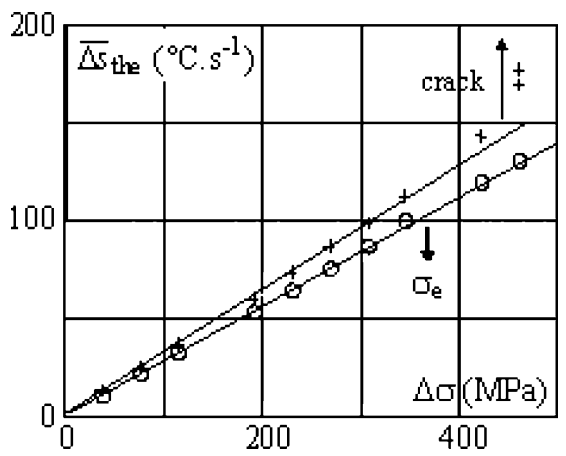

Fig. 11 (empty circle) $\mathrm{min} /$ (positive sign) max values of $\overline{\Delta s}_{\text {the }}$ versus $\Delta \sigma$ induced by the out-of-axis loading 


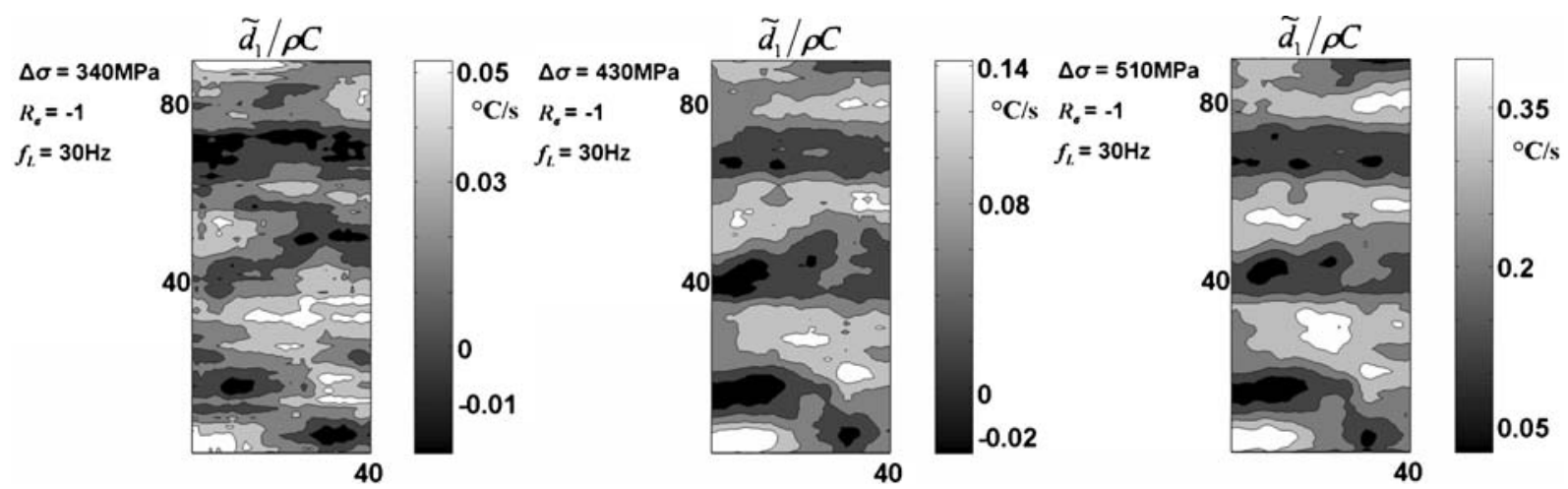

Fig. 12 Dissipation intensity versus stress range

range is representative of the stress pattern, as can be qualitatively observed between Fig. 10(a) and (b).

During the next blocks [Fig. 10(c) and (d)], plasticity develops, thus inducing a stress redistribution. The presence of a stress concentration may explain why the maximal value of $\overline{\Delta s}_{\text {the }}$ is no longer proportional to $\Delta \sigma$. The loss of linearity is naturally less important in regions where stress gradients are lower. In Fig. 10(d), a localization of thermoelastic sources can be observed at the crack tip. The effect of crack propagation at constant stress range on the thermoelastic source can be observed on the right side of Fig. 11.

\section{Properties of $\widetilde{d}_{1}$}

Figure 12 shows the mean dissipation pattern per cycle calculated at different stress ranges. During a loading block, this heterogeneous pattern remains constant as long as a fatigue crack does not initiate.

The contour plots in Fig. 12 also show that areas where the dissipation is high (respectively low) remain approximately the same from one block to another even if the dissipation naturally increases with the stress range. During a (last) loading block, we observed a localized zone of high dissipation several hundreds of cycles before crack onset. Moreover, we also noticed that this zone always corresponds to a candidate zone where the dissipation level is high from the beginning of the fatigue test (i.e. for low stress ranges).

Besides, Fig. 13 shows examples of dissipation variations associated with zones of the same specimen as a function of $\Delta \sigma$ and $f_{\mathrm{L}}$. During this test performed at $R_{\sigma}=-1, \Delta \sigma$ increased from 170 to $500 \mathrm{MPa}$ and for each load level, $f_{\mathrm{L}}$ increased from 9 up to $30 \mathrm{~Hz}$. Dissipation
Fig. 13 Dissipation evolution within two different material zones: (a) "high" intensity zone (b) "low" intensity zone

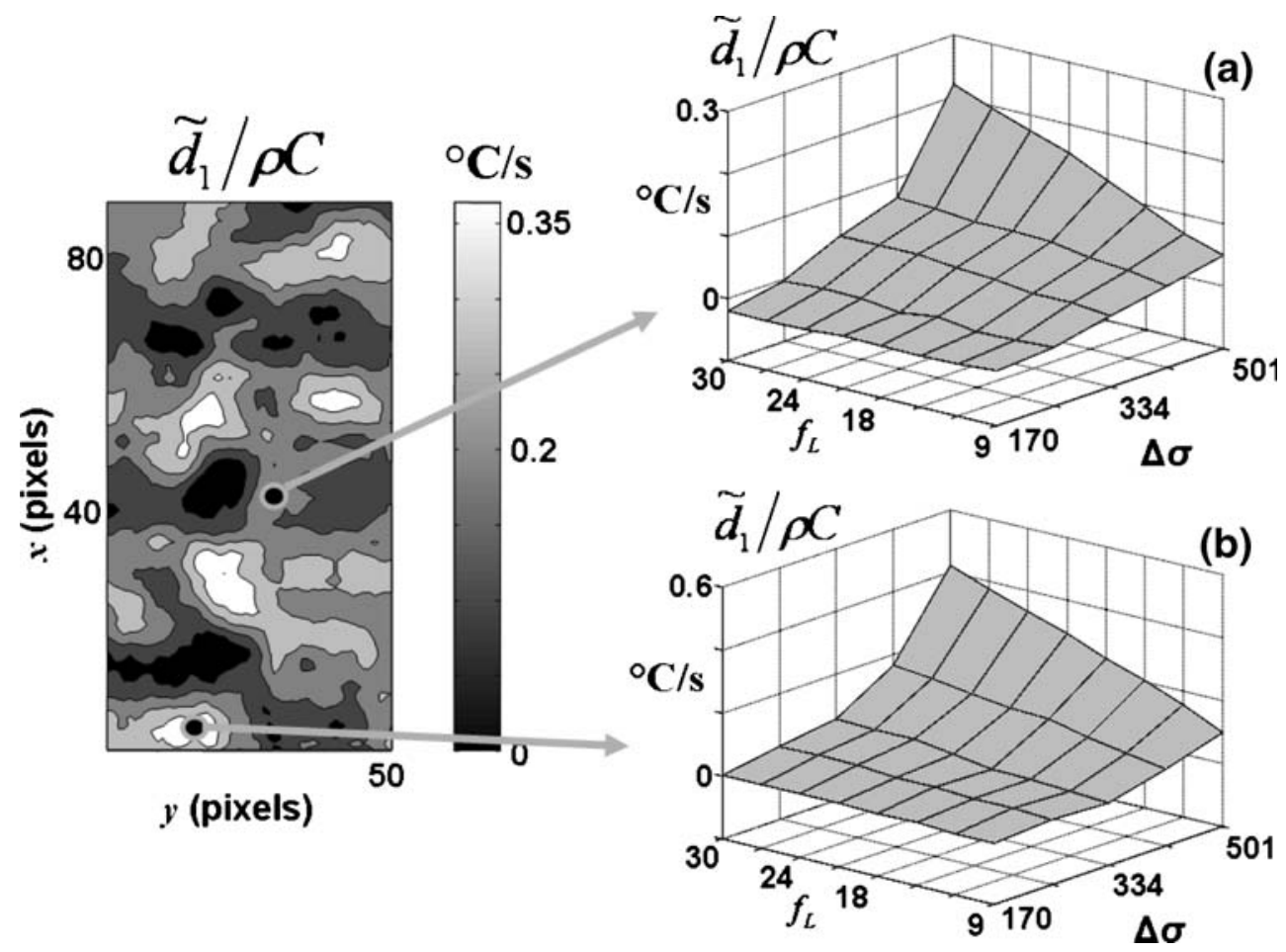


values in the first location [Fig. 13(a)] are twofold lower than that measured in the second area [Fig. 13(b)]. First, it can be noticed that the shape of both surfaces $\widetilde{d}_{1}\left(\Delta \sigma, f_{\mathrm{L}}\right)$ is very similar. At constant stress, we also underline that $\tilde{d}_{1}$ increases in a quasi linear way with the load frequency $f_{\mathrm{L}}$ for both zones and for all stress ranges.

\section{Discussion and Conclusions}

In this paper, an infrared image processing method that enables estimation of coupling and dissipative sources accompanying fatigue was presented. A local expression of the heat equation was thus used. The frequency spectra of the thermoelastic amplitude and of the mean dissipation per cycle allowed us to compute both sources with the same thermosignal. As expected, the sensitivity of the method was closely dependent on the intensity and distribution of the investigated heat sources but also on the signal-to-noise ratio of the temperature measurements. Numerical tests were shown to illustrate the capabilities and limits of the calorimetric approach. In particular, the method failed for very low dissipation levels $\left(<0.03{ }^{\circ} \mathrm{C} \mathrm{s}^{-1}\right)$ and/or for low signal-to-noise ratios.

Applications to Dual Phase 600 steel sheets showed a linear change in the thermoelastic source amplitude as a function of the stress range and the loading frequency, in agreement with the linear thermoelasticity theory. We also observed a heterogeneous dissipation distribution from the beginning of the test. This distribution remained time independent and did not change with the stress range and/or the loading ratio until a fatigue crack onset. Besides, the linear variations in the dissipation intensity with the loading frequency was underlined.

In terms of structure design, this method may be useful for detecting fatigue zones. The thermoelasticity is already used to detect potential crack localisation due to stress concentrations on structures.

In terms of material analysis, obtaining the distribution of thermoelastic and dissipative sources represents precious information for anyone wishing to model fatigue mechanisms. The coupling sources are indeed related to the constitutive state equations while the dissipation is an indicator of the fatigue kinetics and must be associated with the evolution equations (see "Balance of Heat and Definition of Sources" section). Moreover, the dissipation fields enable us to observe the fatigue mechanisms at a finer scale insofar as dissipation is mainly due to the development of persistent slip bands. Finally, calorimetric data are simply new (energy) safeguards for modelling of fatigue mechanisms. Our next objective will be to combine the calorimetric measurement fields with kinematical measurement fields to build up complete, local energy balances as already performed for monotonous loadings in [18]. These energy balances will enable us to derive the internal energy variations induced by high cycle fatigue loading.

Acknowledgements The authors would like to thank Arcelor Research SA and Nippon Steel Corporation for their technical and financial support during this study. They also would like to warmly thank all the members of the Arcelor Research fatigue workgroup for the lively and fruitful discussions.

\section{References}

1. Galtier A (1993) Contribution à l'étude de l'endommagement des aciers sous sollicitations uni ou multiaxiale, ENSAM Thesis.

2. Luong MP (1998) Fatigue limit evaluation of metals using an infrared thermographic technique. Mech Mater 28:155-163.

3. La Rosa G, Risitano A (2002) Thermographic methodology for rapid determination of the fatigue limit of materials and mechanical components. Int J Fatigue 22(1):65-73.

4. Liaw YB, Wang PK, Jiang L, Huang JY, Kuo RC, Huang JC (2001) Thermographic investigation of the fatigue behaviour of reactor pressure vessel steels. Mater Sci Eng A 314:131-139.

5. Krapez JC, Pacou D, Gardette G (2000) Lock-in thermography and fatigue limit of metals. Proceedings of QIRT'2000, Reims, pp 277-282.

6. Krapez JC, Pacou D (2002) Thermography detection of damage initiation during fatigue tests. Proceedings SPIE Thermosense XXIV, Orlando, 4710, pp 435-449.

7. Cugy P, Galtier A (2002) Microplsaticity and temperature increase in low carbon steels. Proceedings 8th Int. Fatigue Conference, Stockholm, pp 549-556.

8. Mabru C, Chrysochoos A (2001) Dissipation et couplages accompagnant la fatigue des matériaux, métalliques. Proceedings of Photomécanique' 01, Poitiers, pp 375-382.

9. Jou D, Casas-Vazquez J, Lebon G (1993) Extended irreversible thermodynamics, Chap. 1. Springer, Berlin, pp 15-39.

10. Capatina A, Stavre R (2000) Algorithms and convergence results for an inverse problem in heat propagation. Int J Eng Sci 38:575587.

11. Rabin Y, Rittel D (2000) Infrared temperature sensing of mechanically loaded specimens: thermal analysis. Exp Mech 40 (2):197-202.

12. Chrysochoos A, Louche H (2000) An infrared image processing to analyse the calorific effects accompanying strain localisation. Int J Eng Sci 38:1759-1788.

13. Dillon OW, Tauchert TR (1966) The experimental technique for observing the temperatures due to the coupled thermoelastic effect. Int J Solids Struct 2(3):385-391.

14. Dillon OW (1966) The heat generated during the torsional oscillations of copper tubes. Int J Solids Struct 2(2):181-204.

15. Boulanger T, Chrysochoos A, Mabru C, Galtier A (2004) Calorimetric and thermoelastic effects associated with the fatigue behavior of steels. Int J Fatigue 26:221-229.

16. Honorat V, Moreau S, Muracciole J-M, Wattrisse B, Chrysochoos A (2005) Calorimetric analysis of polymer behaviour using a pixel calibration of an IRFPA camera. Int $\mathrm{J}$ Quantitative Infrared Thermography 2(2):153-172.

17. Pron H, Bissieux C (2005) Focal plane array infrared cameras as research tools. Int J Quantitative Infrared Thermography 1(2):229-240.

18. Chrysochoos A, Muracciole J-M, Wattrisse B (2000) Experimental analysis of strain and damage localization. Symposium on continuous damage and fracture, Cachan, pp 41-51. 